\title{
Bridging the regeneration gap: insights from echinoderm models
}

In their Review", Sánchez Alvarado and Tsonis present an interesting discussion of “...the model systems that are currently used to dissect the molecular and cellular bases of regeneration, with a focus on in vivo models".

Surprisingly, echinoderms received little attention. Regeneration potential is expressed to a maximum extent in echinoderm $s^{2}$. Larval and adult echinoderms from each of the five classes exhibit natural, rapid regeneration of entire lost parts following predation or other traumatic events (FIG. 1). As adults, echinoderms can regenerate many organs, including limbs, disc, gut, spines and podia and, in some species, regeneration is used for asexual reproduction ${ }^{2,3}$. Moreover, the process has been studied extensively at molecular, cellular, tissue and ecological levels (for example, REFS 2-7).

All the regenerative strategies that are currently described in animals are represented in echinoderms. Arm regeneration in ophiuroids and crinoids is an epimorphic blastemal process, by which new tissues arise from active proliferation of migratory undifferentiated cells (amoebocytes and coelomocytes), which accumulate at the end of the nerve cord as a blastema. In sea stars and sea urchins, morphallaxis is the main regenerative process, involving cells derived from existing tissues by differentiation, transdifferentiation or migration ${ }^{2,3}$. Importantly, echinoderms are deuterostomes and an average of $70 \%$ of echinoderm genes have human homologues ${ }^{8}$. Therefore, the processes involved in echinoderm regeneration are more likely to be extended to mammals than those observed in other classical models such as Hydra or planarians, which are more distantly related to chordates.
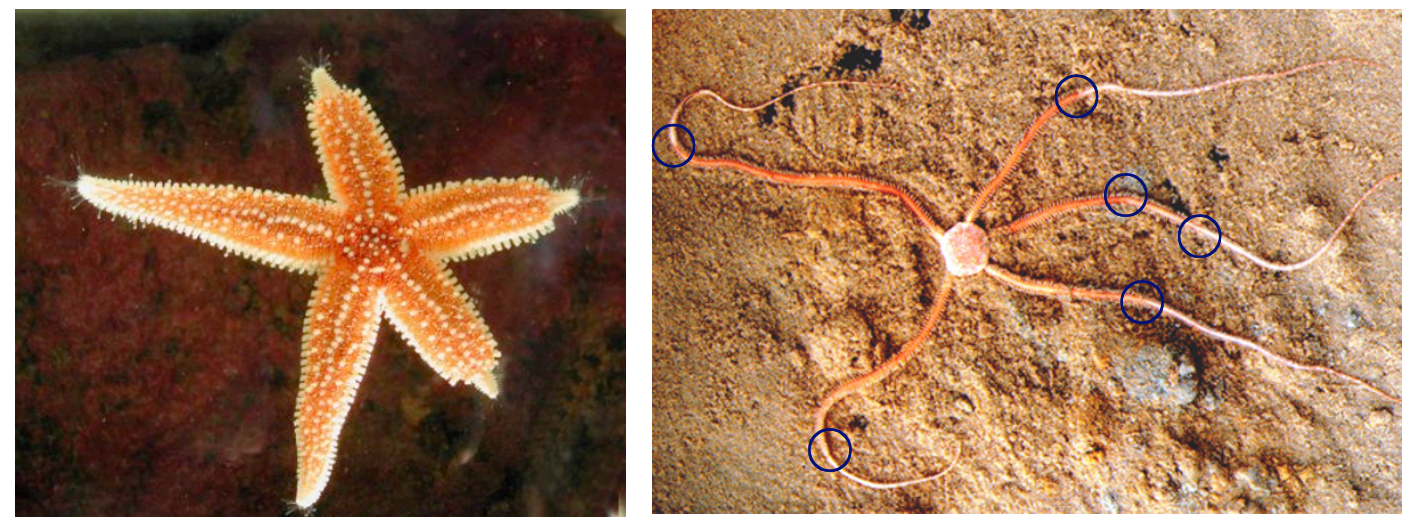

Figure 1 |Two echinoderm models for regeneration studles. a |A sea star, Asterias rubens, with three regenerating arms. b|A brittlestar, Amphiura filiformis, with six scars that indicate sites of regeneration.

Recent studies demonstrate that echinoderms have the potential to offer viable and tractable models for molecular and cellular research on regeneration. First, a growing amount of molecular information about the regeneration process is available. Echinoderms show nerve-dependent regeneration ${ }^{2-7}$, and regeneration in these organisms has been shown to involve growth factors. For example, the bone morphogenetic protein/transforming growth factor- $\beta$ (BMP/TGFB)-signalling pathway has been shown to function in regeneration in brittlestars and crinoids ${ }^{5,9,10}$, the HOX-signalling pathway in brittlestars and seastars ${ }^{6}$, and the Ependymin pathway in the sea cucumber ${ }^{7}$. These molecular pathways are repeatedly encountered during regeneration throughout the animal kingdom ${ }^{1}$. Second, all of the classical cellular and molecular tools are available for these models: EST libraries, not to mention the complete genome of the sea urchin Strongylocentrotus purpuratus ${ }^{8}$, 
immunohistochemistry ${ }^{4,6,9}$, in situ hybridization ${ }^{5,10}$, real-time $\mathrm{PCR}^{7,10}$, microarrays ${ }^{11}$, proteomics and so on. Echinoderm models allow analysis both in vivo and in vitro (for example, cell and tissue explant cultures ${ }^{12}$ ). Finally, Sánchez Alvarado and Tsonis ${ }^{1}$ emphasized the importance of model organisms such as the protostome planarians to ultimately help in the design and implementation of stem-cell therapies in mammals. Echinoderms can be promoted as an alternative deuterostome model in this respect. In a recent study, we have demonstrated that cell proliferation and differentiation rates can be readily manipulated in vivo in the brittlestar Amphiura filiformis, allowing the study of the stem-cell niche during regeneration ${ }^{13}$.

In consequence, echinoderm models have the potential to contribute significantly to the understanding of regeneration at the molecular and cellular levels, are good models for in vivo research on regeneration and, owing to the shared ancestry of echinoderms with chordates, findings from these models are likely to have a positive impact on mammalian regeneration research.

\section{S. Dupont \& M. Thorndyke}

Royal Swedish Academy of Sciences, Kristineberg Marine Research Station, Fiskebackskil, Sweden

1. Sănchez Alvarado, A. \& Tsonis, P. A. Bridging the regeneration gap: genetic insight from diverse animal models. Nature Rev. Genet. 7, 873-883 (2006).

2. Candia Carnevali, M. D. Regeneration in echinoderms: repair, regrowth and cloning. Invert. Surv. J. 3, 64-76 (2006).

3. Thorndyke, M. C., Chen, W., Beesley, P. W. \& Patruno, M. Molecular approach to echinoderm regeneration. Microsc. Res. Tech. 55, 474-485 (2001).

4. Candia Carnevali, M. D. \& Bonasoro, F. Microscopic overview of crinoid regeneration. Microsc. Res. Tech. 55, 403-426 (2001).

5. Bannister, R., McGonnell, I. M., Graham, A., Thorndyke, M. C. \& Beesley, P. W. Afuni, a novel transforming growth factor- $\beta$ gene is involved in arm regeneration by the brittle star Amphiura filiformis. Dev. Genes Evol. 215 , 393401 (2005).

6. Thorndyke, M. C., Patruno, M., Chen, W. C. \& Beesley, P. W. in Brain Stems cells (eds Miyan, J., Thorndyke, M., Beesley, P. W. \& Bannister, C.) 107-120 (BIOS Scientific, Oxford, 2001).

7. Suàrez-Castillo, E. C., Medina-Ortiz, W. E., Roig-Lòpez, J. L. \& GarciaArraras, J. E. Ependymin, a gene involved in regeneration and neuroplasticity in vertebrates, is over expressed during regeneration in the echinoderm Holothuria glaberrima. Gene 334, 133-143 (2004).

8. Sea Urchin Genome Sequencing Consortium. The genome of the sea urchin Strongylocentrotus purpuratus. Science 413, 941-952 (2006).

9. Patruno, M., Smertenko, A., Candia Carnevali, M. D., Bonasoro, F., Beesley, P. W. \& Thorndyke, M. C. Expression of transforming growth factor $\beta$-like molecules in normal and regeneration arms of the crinoid Antedon mediterranea: immunocytochemical and biochemical evidence. Proc. R. Soc. Lond. B Biol. Sci. 269, 1741-1747 (2002).

10. Patruno, M., McGonnell, I., Graham, A., Beesley, P., Candia Carnevali, M. D. \& Thorndyke, M. Anbmp2/4 is a new member of the transforming growth factor- $\beta$ superfamily isolated from a crinoid and involved in regeneration. Proc. R. Soc. Lond. B Biol. Sci. 270, 1341-1347 (2003).

11. Samanta, M. J. et al. The transcriptome of the sea urchin embryo. Science 314, 960-962 (2006).

12. Candia Carnevali, M. D., Bonasoro, F., Patruno, M. \& Thorndyke, M. C. Cellular and molecular mechanisms of arm regeneration in crinoid echinoderms: the potential of arm explants. Dev. Genes Evol. 208, 421-430 (1998). 
13. Dupont, S. \& Thorndyke, M. Growth or differentiation ? Adaptive regeneration in the brittlestar Amphiura filiformis. J. Exp. Biol. 209, 38733881 (2006). 